\title{
Unusually kicked dynamics: Hydrogen atom in a spherical box
}

\author{
Dragoslav R. Mašović ${ }^{*}$ \\ 1 Institute for Nuclear Sciences Vinča, \\ Laboratory for Theoretical and Condensed Matter Physics, \\ 11001 Belgrade, P.O.Box 522, Serbia
}

Received 5 January 2012; accepted 20 April 2012

Abstract:
In this paper we have examined the ionization of the ground state hydrogen atom in a spherical box with
laser pulses of specific shapes. These shapes are predicted assuming correspondence under some condi-
tions with the alternating kicking field. Unusually kicked dynamics is suggested. It is shown that such kicked
dynamics leads to generalized Rabi oscillations with the positive energy states included and participation
of the excited states. The correspondence with the real pulse is established emphasizing such unusually
kicked dynamics. The approach is verified on the one-dimensional (1D) hydrogen atom and calculation of
the known results for ionization probability.

\section{Introduction}

Interest in a hydrogen atom confined within an impenetrable spherical box has been present from the time of the beginning of quantum mechanics to the most recent investigations. During this time many different models of the confined atom with different forms of confinement have been proposed. The atomic nucleus is placed in the center or somewhere off of the center of symmetry of the confining boundary, with different geometric forms and dimensions of the boxes, mainly dependent on the application in the corresponding physical situation [1]. In particular, "the

*E-mail: dmasovic@vinca.rs atom confined in a spherical box" is a useful model for simulating the effect of neighboring atoms in many physical situations. In this manner the interaction of an atom with surrounding atoms, assuming uniformity, is replaced with a sphere in which the considered atom is enclosed [2].

On the other hand, in treating atomic dispersion processes, for example induced by laser fields, the appropriate calculations involve summation over bound eigenstates and integration over unbound eigenstates of the atomic Hamiltonian. The unbound states form a continuum. The treatment of the continuous part of the spectrum is a difficult task and motivates one to seek a method for a discretization of the continuum. Various methods have been proposed (see the short survey in Ref. [3]). The simple version of confinement with the atom placed in the center of a spherical box seems the most appropriate for 
considering dispersion processes and discretization of the continuum. It provides a finite number of wave functions mutually orthogonal and orthogonal to the negative energy states ( ground and excited). Notice that all of the atomic states are now bound by virtue of impenetrable confinement. Thus, the calculation is simplified and estimation of the "continuum" contribution in the process can be obtained. Here we assume that the atomic structure is not disturbed by the laser field.

First, we will give some shortcomings of the one of the utilized methods which includes discretization of the continuum. In the study of ionization of highly excited (Rydberg) atoms by a train of unidirectional or non-alternating electric field pulses, termed half-cycle pulses (HCPs) [4], the numerical solution of the time-dependent Schrödinger equation is based on an expansion of the electron wave function in a finite basis set of Sturmian functions [5]. For an electron in an atom, calculation with a Sturmian basis set with number of basis states $N_{\max }$ assumes solution of the generalized eigenvalue problem with $N_{\max } \times N_{\max }$ matrices and numerical integrations in order to obtain the appropriate matrix elements. The calculation gives a pure discrete spectrum with continuum and bound eigenfunctions. An example is shown in [4] for the continuum, where $N_{\max }=1540$ is used in order to obtain agreement with the exact wave function over the box with the radius $\mathrm{R}=300$ a.u. Furthermore, the Sturmian functions depend on the free parameter $\lambda$ (each value of $\lambda$ provides a different complete set). Thus, the questions are: (i) how large should the basis set be, and (ii) what value should $\lambda$ take in order to get an accurate result with the smallest basis set. The answers are given in [4] but in general they are accomplished with a lot of tests and need high performance computers.

The problem of an atom subject to a train of HCPs pulses whose duration is short compared with the orbital period of a classical electron (Rydberg states) are extensively treated both experimentally and theoretically [6]. $\delta$-shaped pulses (kicks) are a widely used approximation (the sudden approximation [4]) for the electric filed.

A kicked hydrogen atom initially in the ground state is considered in Ref. [7]. Here, the one half-period of the electric field is substituted by a single $\delta$ kick resulting in an alternating kicking pulse and in this approximation ionization in an ultra strong laser filed is considered. In the a recent paper [8] the ionization probability for a hydrogen atom in the limit of very short electric field pulses is investigated. It is shown that for a one-cycle pulse the ionization caused by the first half-cycle is reversed by the second (recombination occurs), giving Rabi flopping involving the continuum. Thus, in this paper we explore the possibility for generalized Rabi oscillations with the positive energy states included and participation of the excited states during the total pulse duration assuming specific, proposed shapes of laser pulses on the basis of established kick dynamics.

The atomic units $\hbar=\mu=e=1$ are used through the paper, where $\hbar$ is Planck's contant and $\mu$ and $e$ are the electron mass and charge, respectively.

The organization of the rest of the paper is as follows. In Sec. 2 the model of a hydrogen atom in a spherical box is presented. In Sec. 3 we consider the ionization of a ground state hydrogen atom. The verification of the method is given in Sec. 4 and the conclusions in Sec. 5 .

\section{Hydrogen atom in spherical box}

The simplest way to introduce discretization in the continuum assumes the strong field approximation (SFA) and the free spherical waves [9]:

$$
\Psi_{k l m}(r, \theta, \varphi)=R_{k l}(r) Y_{l}^{m}(\theta, \varphi)=\sqrt{\frac{2 k^{2}}{\pi}} j_{l}(k r) Y_{l}^{m}(\theta, \varphi),
$$

where $l$ and $m$ are the orbital angular and magnetic quantum numbers, respectively and $(r, \theta, \varphi)$ are spherical polar coordinates. The radial part $j_{l}$ is the spherical Bessel function and the spherical harmonics $Y_{l}^{m}$ provide the angular part of the wave functions. $k$ is given by $E=\frac{\hbar^{2} k^{2}}{2 \mu}$, where $E$ is the electron energy. The impenetrable spherical box of the radius $a$ imposes the boundary condition

$$
j_{l}(k a)=0,
$$

and as solutions the set $\left\{k_{n l}\right\}, n=1,2,3 \ldots$ is obtained for the given $l$ value.

In framework of the SFA it is assumed that we have a free electron with well-defined momentum $\hbar k$ and the Coulomb interaction with the parent ion is completely neglected. But the effect of the Coulomb potential has many manifestations in ionization of hydrogen atom irradiated by an intense laser field [10]. Thus, it is more reasonable to suppose instead of (1) the exact continuum function [11]

$$
\begin{array}{r}
R_{k l}(r)=\sqrt{\frac{2}{\pi}} e^{\frac{\pi Z}{2 k}} \frac{\left|\Gamma\left(l+1-i \frac{Z}{k}\right)\right|}{(2 l+1) !}(2 k r)^{l} e^{-i k r} \\
M\left(1+l+i \frac{Z}{k}, 2 l+2,2 i k r\right),
\end{array}
$$

where for hydrogen $Z=1, M$ is the confluent hypergeometric function and $k>0$. 
In the spherical box with the nucleus in the center we can take (3) in the form

$$
u_{k l}(r)=r R_{k l}(r)=C_{k l} F_{l}\left(-\frac{Z}{k}, k r\right),
$$

where $F_{l}$ is the regular Coulomb wave function and $C_{k l}$ is the normalization constant. We use the COULFG program for calculation the regular Coulomb wave function with different values of the parameters ACCUR and ABORT than the ones given in [12]. Note that the most recent program for computing hydrogenic continuum wave functions is now available [13].

Assuming that the radius $a$ is large enough, we obtain the same low energy part of the negative spectrum as for the free hydrogen atom. For the positive energy states, the set $\left\{k_{n}\right\}$ are the solutions of the following equation

$$
F_{l}\left(-\frac{Z}{k}, k a\right)=0
$$

in the range $\left[k_{\min }, k_{\max }\right]$ for the given $l$. The low energy part of the positive energy states corresponding to $\left[0, k_{\min }\right]$ is neglected, and, similarly, the Sturmian basis is not adequate for describing in this range [14].

For $k \rightarrow 0$ the limiting form of $F_{l}\left(-\frac{z}{k}, k a\right)$ in (5) must be considered [15]. In order to avoid it, an estimation for $k_{\min }$ is required and $E_{\min } \geq 5 \cdot 10^{-9}$ a.u. was suggested in [12]. Thus, we have tested our modified program down to $E_{\text {min }} \sim 10^{-10}$ a.u. Similarly, the limit $k a \rightarrow \infty$ assumes an asymptotic expansion for $F_{l}\left(-\frac{z}{k}, k a\right)$ and therefore the condition $k_{\max } a \leq 10^{4}$ is imposed in [12].

Here, we assume that $E_{\text {min }}=0.00125$ a.u. and $E_{\text {max }}=$ 0.3 a.u. are quite enough for further considerations. In a following Section we check our estimation.

Additionally, expansion of $u_{k l}(r)$ in terms of Bessel functions [16]:

$$
u_{k l}(r)=C \sum_{i=l}^{\infty} b_{i}(k) \sqrt{r} J_{i+\frac{1}{2}}(k r),
$$

can be very useful in applications, assuming finite $k r$. Such kinds of expansion are also attractive for mathematicians $[15,17]$. Similarly, expansion of the continuum functions in terms of a Laguerre basis starting from the paper of Yamani and Reinhardt [18] have been the subject of a considerable number of studies (see for example [19, 20]). Now, completeness assumes

$$
\sum_{j}|j\rangle\langle j| \approx 1
$$

where in the sum both negative and positive energy states of the atomic Hamiltonian are included. In (7) the upper part of excited states and the lower part of positive energy states are neglected. In recent papers [21, 22], an atom in a cavity is of interest with respect to the electron scattering. In particular, for a hydrogen atom [22] s-wave scattering is considered and the phase shift is given by

$$
\delta_{0}^{\alpha}=-k_{\alpha} a+\alpha \pi
$$

where the $k_{\alpha}$ are obtained calculating positive orbital energies for the given $a$.

In our case in the limit $\rho=k r \rightarrow \infty$

$$
F_{l} \sim \sin \left(\rho-l \frac{\pi}{2}-\eta \ln 2 \rho+\sigma_{l}\right)
$$

where $\sigma_{l}$ is the Coulomb phase shift [16]. Thus, we obtain for partial phase shift

$$
\delta_{l}=-\eta \ln 2 \rho+\sigma_{l}
$$

For $l=0 \Rightarrow \delta_{0}=\delta_{0}\left(k_{n 0}\right)=\delta_{0}^{n 0}$ in (10), where $k_{n 0}$ are the solutions of (5), formulas (10) and (8) are in agreement assuming $k_{n 0} a \rightarrow \infty$.

\section{Ionization of hydrogen atom}

The ionization of the ground state of the hydrogen atom by an electric field pulse in sinusoidal form featured through a sine-squared envelope is a well-known problem. Assumption of a spherical box and discretization of continuum was first performed in [23] and then in [24, 25]. Within the framework of this approach only the positive energy wave functions which vanish at the end of chosen box are distinguished and normalized. Then, the eigendifferential method [26] is applied and they are grouped into smaller sets. From each set the resulting basis function is formed [24]

$$
\bar{u}_{k_{i} l}(r)=\frac{1}{\sqrt{2 N+1}} \sum_{j=i-N}^{i+N} u_{k_{j} l}(r)
$$

and included in expansion. The advantage of such coarse graining is that the number of basis set wave functions is reduced by a factor $2 N+1$ while the boundary condition is maintained.

Now, we will consider a hydrogen atom in an alternating kicking field

$$
F(t)=F_{0} T\left[\delta\left(t-\frac{T}{4}\right)-\delta\left(t-\frac{3 T}{4}\right)\right]
$$


for $0 \leq t \leq T$, where $F_{0}$ is the strength of the field in the $z$ direction, $T$ is the period, and $\omega=\frac{2 \pi}{T}$ is the angular frequency. The one-period dynamics is given by evolution operator

$$
U(T)=e^{-\frac{i}{\hbar} H_{0} \frac{T}{4}} e^{\frac{i}{\hbar} \Delta p z} e^{-\frac{i}{\hbar} H_{0} \frac{T}{2}} e^{-\frac{i}{\hbar} \Delta p z} e^{-\frac{i}{\hbar} H_{0} \frac{T}{4}},
$$

where $\Delta p=e F_{0} T$ is the kick strength. The transition probability is

$$
P_{i f}(T)=|\langle f|U(T)| i\rangle|^{2},
$$

where $i$ is the initial (1s) and $f$ the final state of atomic Hamiltonian. The ionization probability is

$$
P_{\text {ion }}=1-\sum_{b} P_{i b}
$$

where $b$ corresponds to the negative energy states.

In order to calculate (14) and (15) we use (6) and obtain

$$
P_{i f}(T)=\left|\sum_{j} e^{-i \frac{T E_{j}}{2 \hbar}}\left\langle f\left|e^{i \frac{\Delta p}{\hbar} z}\right| j\right\rangle\left\langle j\left|e^{-i \frac{\Delta p}{\hbar} z}\right| i\right\rangle\right|^{2}
$$

where $E_{j}$ is the electron energy. In (16) for the magnetic quantum number $m=0$ is used and it is constant with respect to the cylindrical symmetry in the field. Now, we are in the position to estimate all possible negativenegative, negative-positive and positive-positive contributions in (16) assuming two different values $a=200$ and 1000 a.u.

In order to determine the low part of the negative energy states for the given value $a$ we compare the following integrals

$$
l_{1}=\int_{0}^{a} F_{n l}^{2}(r) d r \quad I_{2}=\int_{0}^{2 a} F_{n l}^{2}(r) d r,
$$

where

$$
F_{n l}(r)=e^{-\frac{Z r}{n}} r^{l+1} L_{n-l-1}^{2 l+1}\left(\frac{2 Z r}{n}\right)
$$

corresponds to the exact unnormalized hydrogenic radial function, $n$ is the principal quantum number, and $L_{n-l-1}^{2 l+1}$ are the associated Laguerre polynomials. Only $l=0,1$ and 2 values are considered. Then, for $a=200$ we obtain the following results

$$
\begin{array}{rll}
1 \leq n \leq 6 & \rightarrow & I_{1}=I_{2} \\
n=7 & \rightarrow & I_{1} \cong I_{2} \\
7<n \leq 10 & \rightarrow & l_{1} \approx I_{2},
\end{array}
$$

and for $a=1000$

$$
1 \leq n \leq 18 \quad \rightarrow \quad l_{1}=I_{2} \quad \text { or } \quad l_{1} \cong I_{2}
$$

It means that use of the max $n=10$ is acceptable for both of the $a$ values.

Thus, (16) may be written as

$$
\begin{aligned}
P_{i f}(T)= & \mid \sum_{b} e^{-i \frac{T E_{b}}{2 \hbar}}\left\langle f\left|A^{+}\right| b\right\rangle\langle b|A| i\rangle+ \\
& \left.\sum_{c} e^{-i \frac{T E_{c}}{2 \hbar}}\left\langle f\left|A^{+}\right| c\right\rangle\langle c|A| i\rangle\right|^{2},
\end{aligned}
$$

where $A=e^{-i \frac{\Delta p}{\hbar} z}$ and $c$ corresponds to the positive energy states. First, for $f \rightarrow c$, we compare

$$
P_{i c}^{1}(T)=\left|\sum_{b} e^{-i \frac{T E_{b}}{2 \hbar}}\left\langle c\left|A^{+}\right| b\right\rangle\langle b|A| i\rangle\right|^{2},
$$

and

$$
P_{i c}^{2}(T)=\left|\sum_{c} e^{-i \frac{T E_{c}}{2 \hbar}}\left\langle c\left|A^{+}\right| c\langle\rangle c|A| i\right\rangle\right|^{2} .
$$

It gives $P_{i c}^{2}=O\left(P_{i c}^{1}\right)$ and the positive-positive part is neglected. Similarly, for $f \rightarrow b$, we compare

$$
\begin{aligned}
P_{i b}^{1}(T)= & \mid \sum_{b} e^{-i \frac{T E_{b}}{2 \hbar}}\left\langle b\left|A^{+}\right| b\right\rangle\langle b|A| i\rangle+ \\
& \left.\sum_{c} e^{-i \frac{T E_{c}}{2 \hbar}}\left\langle b\left|A^{+}\right| c\right\rangle\langle c|A| i\rangle\right|^{2},
\end{aligned}
$$

and

$$
P_{i b}^{2}(T)=\left|\sum_{b} e^{-i \frac{T E_{b}}{2 \hbar}}\left\langle b\left|A^{+}\right| b\right\rangle\langle b|A| i\rangle\right|^{2} .
$$

The difference is neglected and we can definitively accept for completeness

$$
\sum_{b}|b\rangle\langle b| \sim 1
$$

as a reasonable approximation.

We calculated the transition probability $P_{i b}(T)$ assuming (24) for a few values of $\omega$ and $F_{0}$. We always obtain the maximum of $P_{i b}(T)$ for the ground state. It is decreasing function of $n$. This is also confirmed in the most extensively examined example where $P_{i b}(T)$ is calculated according to (7) for $a=1000$ a.u., $1 \leq n \leq 12$, $\omega=0.375$ a.u., and $F_{0}=0.02$ a.u. Thus, we concluded 
that for the low part of the negative energy states we can assume that only the free hydrogen atom states with $1 \leq n \leq 10$ and $l=0,1,2$ are included in (24).

In the previous example we tested our estimation for $E_{\text {min }}$ and $E_{\max }$ from Sec. 2. In this case the total probability is calculated according to

$$
P_{\text {tot }}=\sum_{b} P_{i b}(T)+\sum_{c} P_{i c}(T) .
$$

The first term in (25) gives 0.903 for probability. Notice that the second term assumes summations over $l$ (up to 2 ) and allowed $k$ values in $\left[k_{\min }, k_{\max }\right]$. The calculation of this term needs extensive computational time and therefore it is restricted to the segments of allowed $k$ values in $\left[k_{\min }, k_{\max }\right]$ with distinct probabilities. Thus, we obtain $P_{\text {tot }}=0.97$ which is enough for confirmation. Further inclusion of $k<k_{\min }$ and $k>k_{\max }$ seems unnecessary. In order to calculate the probabilities for $U(2 T), U(3 T), \ldots$ the inclusion of (24) between the exponential operators is multiplied and after $3 T$ calculation is virtually impossible for us, and needs enormous computational time. Therefore, a different approach is appropriate. Assuming

$$
\left|\psi^{1}\right\rangle=U(T)|i\rangle
$$

then

$$
\left|\psi^{2}\right\rangle=U(T)\left|\psi^{1}\right\rangle
$$

is obtained from the known $\left\langle b \mid \psi^{1}\right\rangle=\psi_{b}^{1}$ and so on, similarly as in Ref. [27] for the exponential split operator method. The computational time is drastically reduced and the results are in the full agreement with the previous. However, in either case the closure (24) is always present and the question arises as to how we can estimate the accuracy of the results.

\section{Test 1}

We consider (13) introducing an arbitrary time delay $\varphi_{0}$ between the kicks. Then, the evolution operator is

$$
U(1)=e^{-\frac{i}{\hbar} H_{0} \frac{T}{4}} e^{\frac{i}{\hbar} p z} e^{-\frac{i}{\hbar} H_{0}\left(\frac{T}{2}+\varphi_{0}\right)} e^{-\frac{i}{\hbar} p z} e^{-\frac{i}{\hbar} H_{0} \frac{T}{4}},
$$

where $p=e F_{0} T$. It can be simply shown that for $\varphi_{0}=-\frac{T}{2}$ we must have $P_{i o n}=0$ assuming "ideal" completeness. But, for $\omega=0.6$ a.u. we obtain the following results:

$$
\begin{array}{llll}
F_{0}=0.04 \text { a.u. } & \rightarrow & P_{\text {ion }}=0.10 \\
F_{0}=0.08 \text { a.u. } & \rightarrow & P_{\text {ion }}=0.42
\end{array}
$$

as "zero" values. The results are confirmed for $a=$ 1000 a.u. and maximum value of the principal quantum number $n=14$. It means that any changes after oneperiod dynamics less or equal than "zero" are meaningless. For example, for $F_{0}=0.04$ a.u. we calculated $P_{\text {ion }}$ for $\varphi_{0}=-\frac{T}{8}, \frac{T}{4}$ and $\frac{T}{2}$. Thus, we obtain $0.16,0.21$ and 0.19 , respectively. All the results are above the "zero" value.

\section{Test 2}

We examine the $U(2)$ dynamics in a similar way introducing arbitrary time delays $\varphi_{1}$ and $\xi_{1}$. Therefore,

$$
\begin{aligned}
U(2)= & e^{-\frac{i}{\hbar} H_{0} \frac{T}{4}} e^{\frac{i}{\hbar} p z} e^{-\frac{i}{\hbar} H_{0}\left(\frac{T}{2}+\xi_{1}\right)} e^{-\frac{i}{\hbar} p z} e^{-\frac{i}{\hbar} H_{0}\left(\frac{T}{4}+\varphi_{1}\right)} \\
& e^{-\frac{i}{\hbar} H_{0} \frac{T}{4}} e^{\frac{i}{\hbar} p z} e^{-\frac{i}{\hbar} H_{0} \frac{T}{2}} e^{-\frac{i}{\hbar} p z} e^{-\frac{i}{\hbar} H_{0} \frac{T}{4}}
\end{aligned}
$$

and taking $\varphi_{1}=-\frac{T}{2},(29)$ is reduced to

$$
U(2)=e^{-\frac{i}{\hbar} H_{0} \frac{T}{4}} e^{\frac{i}{\hbar} p z} e^{-\frac{i}{\hbar} H_{0}\left(\frac{T}{2}+\xi_{1}\right)} e^{-\frac{i}{\hbar} H_{0} \frac{T}{2}} e^{-\frac{i}{\hbar} p z} e^{-\frac{i}{\hbar} H_{0} \frac{T}{4}}
$$

and for $\xi_{1}=-T$ to

$$
U(2)=e^{-\frac{i}{\hbar} H_{0} \frac{T}{2}}
$$

The expectation is $P_{\text {ion }}=0$, but in calculation for $\omega=$ $0.6 a$.u. and $F_{0}=0.04 a$.u. we obtain $P_{\text {ion }}=0.30$. This is the "zero" value for two-period dynamics. Evidently, with multiplicative application of (3.14) the error is increased. We calculated the ionization probability for many different, physical acceptable values $\varphi_{1}$ and $\xi_{1}$. The changes are always inside of "zero" value. Thus, it is desirable to stay in essentially one-period dynamics.

The results in Test 2 have been checked with calculations based on two different approaches. Full agreement is always present.

Let us consider

$$
\bar{U}(n T)=e^{-\frac{i}{\hbar} H_{0} \frac{n T}{4}} e^{\frac{i}{\hbar} \Delta p z} e^{-\frac{i}{\hbar} H_{0} \frac{n T}{2}} e^{-\frac{i}{\hbar} \Delta p z} e^{-\frac{i}{\hbar} H_{0} \frac{n T}{4}} .
$$

We obtain (32) by replacing in (13) $T \rightarrow n T$ and $F_{0} \rightarrow$ $\frac{F_{0}}{n} \quad \Rightarrow \Delta p=e F_{0} T=$ const.

Then, for the transition probability in (14) we have $P_{i f}(T) \rightarrow P_{i f}(n T)$.

How do the dynamics look according to $\bar{U}(n T)$ ?

Suppose that we have an unknown (for now) pulse with an electric field $E(t)$. Then, for $n=1, \bar{U}(T)$ recognizes it as in (12) during the time $[0, T]$ assuming

$$
\frac{1}{T} \int_{0}^{\frac{T}{2}} E(t) d t=F_{0}, \quad \frac{1}{T} \int_{\frac{T}{2}}^{T} E(t) d t=-F_{0}
$$


Looking at $n=2, T \rightarrow 2 T, F_{0} \rightarrow \frac{F_{0}}{2}$, and (12) becomes

$$
F(t)=F_{0} T\left[\delta\left(t-\frac{T}{2}\right)-\delta\left(t-\frac{3 T}{2}\right)\right] .
$$

This means $\bar{U}(2 T)$ recognizes the unknown pulse as in (34) during the time $[0,2 T]$ assuming

$$
\frac{1}{2 T} \int_{0}^{T} E(t) d t=\frac{F_{0}}{2}, \quad \frac{1}{2 T} \int_{T}^{2 T} E(t) d t=-\frac{F_{0}}{2}
$$

and so on. We will determine the unknown pulse from the conditions (33), (35),...

Let the total pulse duration be $2 \tau$ and $E(t)$ defined in the interval $(-\tau, \tau)$. Then, the expansion in a Fourier series is

$$
E(t)=\frac{a_{0}}{2}+\sum_{n=1}^{N}\left(a_{n} \cos \frac{\pi n t}{\tau}+b_{n} \sin \frac{\pi n t}{\tau}\right) .
$$

It satisfies [28]

$$
\int_{-\tau}^{\tau} E(t) d t=0
$$

giving $a_{0}=0$. We have $E(-t)=-E(-t) \Rightarrow a_{n}=0$ and

$$
E(-\tau)=E(\tau)=0
$$

at the ends of the pulse. Note, from (33) it follows that

$$
\frac{1}{T} \int_{0}^{T} E(t) d t=0
$$

contrary to the condition in (35). Therefore, to avoid contradictions we modified (33) and (35)

$$
\begin{gathered}
\frac{1}{T} \int_{0}^{\frac{T}{2}+\varepsilon} E(t) d t=F_{0} \quad \frac{1}{T} \int_{\frac{T}{2}+\varepsilon}^{T} E(t) d t=-F_{0} \\
\frac{1}{2 T} \int_{0}^{T+\varepsilon} E(t) d t=\frac{F_{0}}{2} \quad \frac{1}{2 T} \int_{T+\varepsilon}^{2 T} E(t) d t=-\frac{F_{0}}{2},
\end{gathered}
$$

with $\varepsilon$ small. Assuming for the pulse $\tau=M T$, the problem is reduced to the solution of $\mathrm{N} \times \mathrm{N}$ linear equations, where $N=2 M$.

Thus, the solutions $b_{n}$ in (3.26) depend on the parameter $\varepsilon$. We examined the $\varepsilon$ dependence for $M=2, F_{0}=0.04$ a.u., $\omega=0.6$ a.u. and the values $\varepsilon=0.01,0.1,0.5,1$ and 2 a.u. A remarkable decrease of $E(t)$ is visible: from max $E(t) \sim$ 60 a.u.( $(\varepsilon=0.01$ a.u.) to $\max E(t) \sim 0.3 a . u .(\varepsilon=2 a . u$. $)$. For example, the result is shown in Fig. 1 for $\varepsilon=0.1$ a.u.

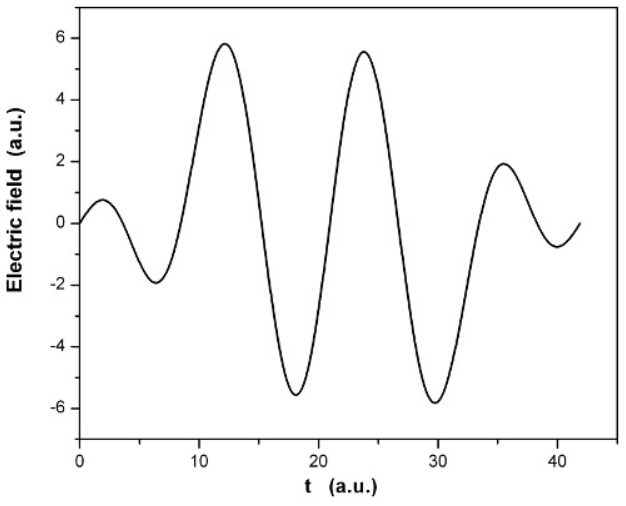

Figure 1. The form of the pulse for $\omega=0.6$ a.u., $F_{0}=0.04$ a.u., $M=2$ and $\varepsilon=0.1$ a.u.

The total pulse duration is $1 \mathrm{fs}$ and femtosecond pulse shaping is possible [29].

We calculated the ionization probability $P_{\text {ion }}(n T)$ assuming kick dynamics $U(n T)$ in (32) for $F_{0}=0.04$ and 0.08 a.u. The results are shown in Fig. 2 and Fig. 3, respectively, and they are above "zero" values. The corresponding fits are in the form

$$
P_{\text {ion }}=c_{1}+c_{2} \cdot \sin ^{2}\left(\frac{\omega_{R}}{2} t\right)
$$

where

$$
\omega_{R} \sim \frac{0.99}{\pi} \omega
$$

providing the evidence for what we have called generalized Rabi oscillations. It seems more appropriate than "Rabi flopping involving the continuum" [8] since here, besides inclusion of the positive energy states, the excited states also participate in the process.

To support this assertion we calculated the transition probability $P_{\text {if }}$ for some of the $t / T$ values assuming $f \rightarrow c$. The results for $a=200$ a.u. are shown in Fig. 4 and Fig. 5 for orbital quantum numbers $l=1$ and 2 , respectively. They show the probability for one-photon absorption ( $\hbar \omega=16.3 \mathrm{eV}$ ) as the minimum required for ionization. The oscillations are clearly visible. The results in Figs. 4 and 5 were checked for $a=1000$ a.u. The shapes of the probability functions are unchanged, meaning that the results are independent of $a$.

\section{Verification of the method}

In order to verify the method we consider a 1D hydrogen atom in the external field $E(t)$ given by (36). The 


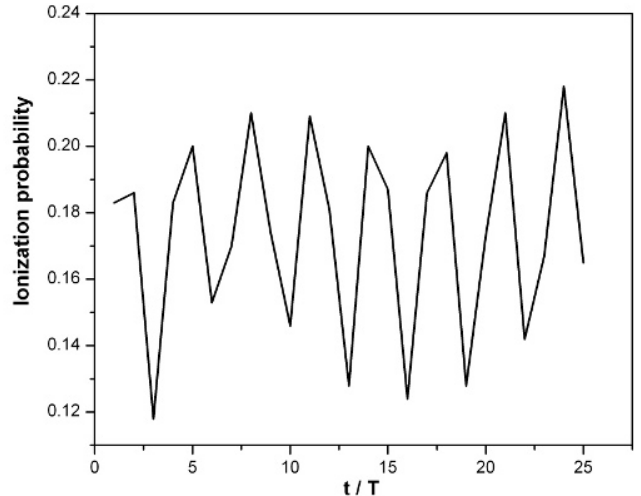

Figure 2. Ionization probability for $\omega=0.6$ a.u. and $F_{0}=0.04$ a.u.

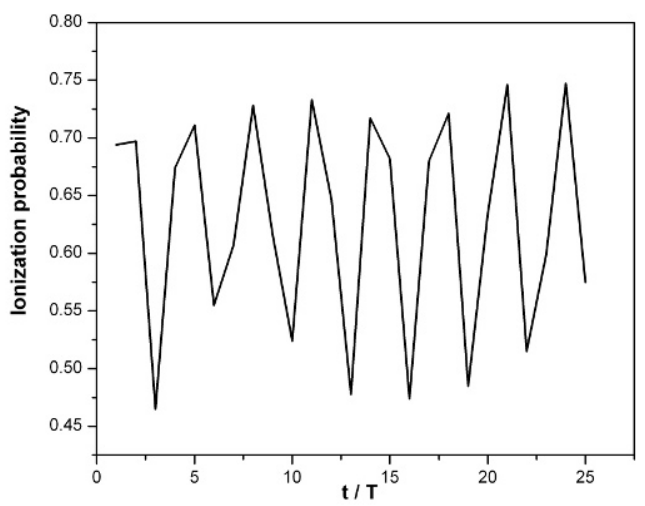

Figure 3. Ionization probability for $\omega=0.6$ a.u. and $F_{0}=0.08$ a.u.

appropriate Hamiltonian in the length gauge is

$$
H=-\frac{1}{2} \frac{\partial^{2}}{\partial x^{2}}+V(x)+x E(t)=H_{0}+x E(t)
$$

where $H_{0}$ is the "unperturbed" part and for $V(x)$ we take soft-core potential [30]

$$
V(x)=-\frac{1}{\sqrt{1+x^{2}}}
$$

with impenetrable box conditions at $x=-a$ and $x=a$. We use the first-order sympletic scheme [31] to find the eigenvalues $E_{0}, E_{1}, E_{2}, \ldots$ of $H_{0}$ and the corresponding eigenvectors. Assumption of the soft-core potential

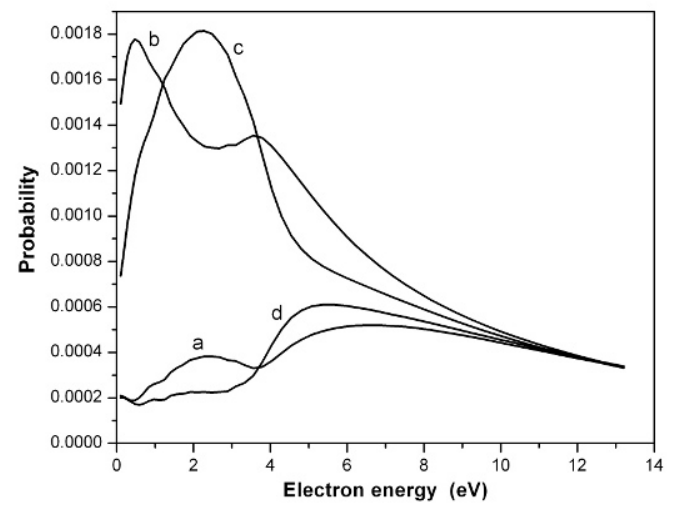

Figure 4. Transition probability $P_{i f}, f$ corresponds to the positive energy states; $l=1, \omega=0.6$ a.u., $F_{0}=0.04$ a.u. and $a=200$ a.u.; a $\frac{t}{T}=13 \quad P_{\text {ion }}=0.128 ; \quad$ b $\quad \frac{t}{T}=$ $14 P_{\text {ion }}=0.200 ; \mathrm{c} \quad \frac{t}{T}=15 \quad P_{\text {ion }}=0.187 ; \quad \mathrm{d}$ $\frac{t}{T}=16 \quad P_{\text {ion }}=0.124$

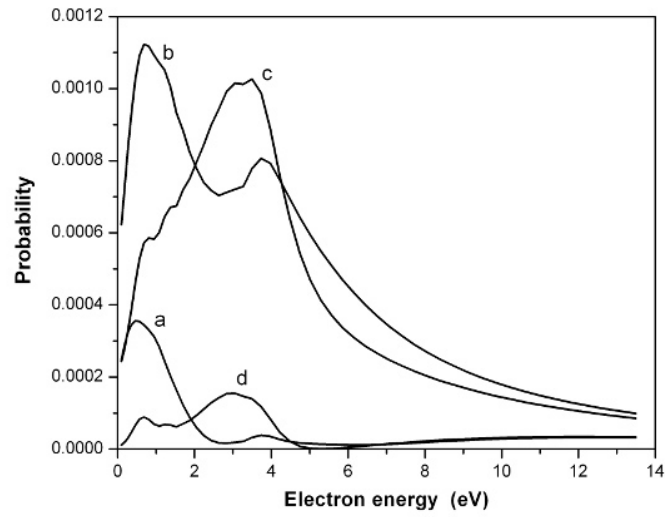

Figure 5. Transition probability $P_{i f}, f$ corresponds to the positive energy states; $l=2, \omega=0.6$ a.u., $F_{0}=0.04$ a.u. and $a=200$ a.u. For a, b, c, and d the same as in Fig. 4.

in (45) has the advantage that parity is a good quantum number. Thus, the eigenstates so obtained can be either even or odd. A grid of $x$ points with $\Delta x=a / 1000$, where $a=100$ a.u., makes it possible to calculate the energies $E$ given in Table I in Ref. [30] with the same accuracy. Then, with the time step $\Delta t=0.08$ a.u. the appropriate wave functions assuming the interaction term in (44) are carried out according to the standard Crank-Nicholson al- 


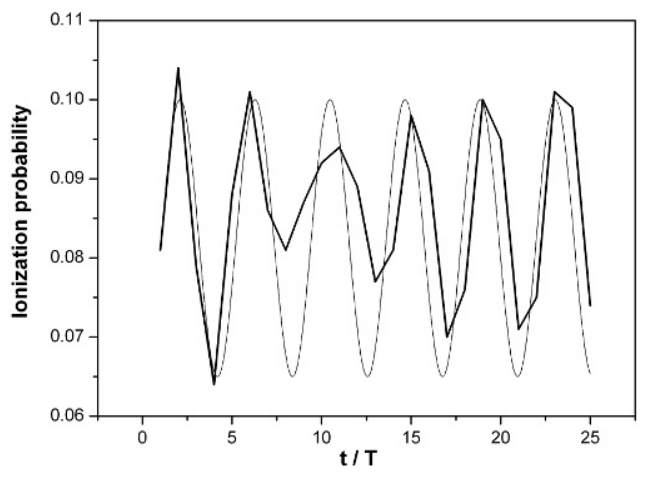

Figure 6. Ionization probability for $\omega=0.8$ a.u. and $F_{0}=0.04$ a.u. The fit is also shown.

gorithm [30]

$$
\begin{aligned}
\psi(t+\Delta t)= & {\left[1-\frac{i \Delta t}{2} H\left(t+\frac{\Delta t}{2}\right)\right] } \\
& {\left[1+\frac{i \Delta t}{2} H\left(t+\frac{\Delta t}{2}\right)\right]^{-1} \psi(t), }
\end{aligned}
$$

from $t=0$ up to the desired time.

In the following we will assume $\omega=0.8$ a.u. for the one-photon absorption,

$$
E_{i}+\omega=0.1302 \text { a.u., }
$$

and the hydrogen atom is initially in the ground state $i$. Then we calculated the ionization probability $P_{i o n}(n T)$ with kick dynamics $\bar{U}(n T)$ for $F_{0}=0.04$ a.u. The results are shown in Fig. 6 and they are above the "zero" value 0.055. The appropriate angular Rabi frequency is

$$
\omega_{R} \sim \frac{0.75}{\pi} \omega
$$

and $T_{R} \sim 32.9$ a.u. For the electric field pulse we take $M=2$ and the total pulse duration is $2 \tau=31.4$ a.u. Thus, we can estimate

$$
T_{R} \sim 2 \tau \sim 32 \text { a.u. }
$$

This can be useful in order to follow oscillations in the probability.

First, we examined the case where the parameter $\varepsilon=$ 0.1 a.u. The form of the pulse is similar to that in Fig. 1. The obtained results for the probability

$$
P_{i i}(t)=\left|\left\langle i \mid \psi_{i}(t)\right\rangle\right|^{2}
$$

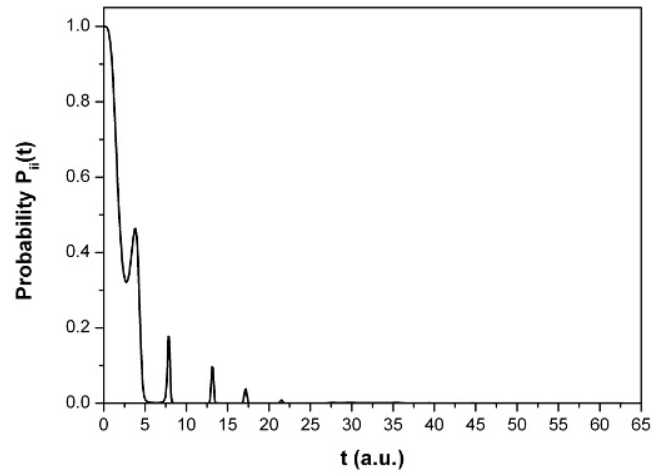

Figure 7. Dependence of probability $P_{i i}(t)$ for pulse with $\omega=$ 0.8 a.u. and $\varepsilon=0.1$ a.u.

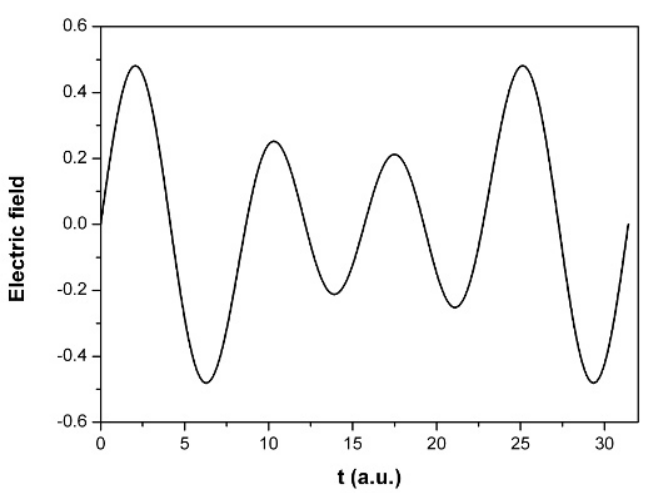

Figure 8. The form of the pulse for $\omega=0.8$ a.u., $F_{0}=0.04$ a.u., $M=2$ and $\varepsilon=3$ a.u.

are shown in Fig. 7. It is obvious that there are no oscillations in either $P_{i i}(t)$ or in the ionization probability, assuming a pulse with ultra strong electric field.

However, let us consider the case with $\varepsilon=3$ a.u. The appropriate form of the pulse is shown in Fig. 8 and the results for $1-P_{i i}(t)$ are given in Fig. 9. Now we have almost symmetrical behaviour in $1-P_{i i}(t)$ for $t \sim T_{R}$. The ionization probability is calculated according to

$$
P_{\text {ion }}(t) \sim 1-\left[P_{i i}(t)+\sum_{n=1}^{N} P_{i n}(t)\right]
$$

where

$$
P_{i n}(t)=\left|\left\langle i \mid \psi_{n}(t)\right\rangle\right|^{2}
$$

and assuming a finite number of excited states $N$ in (51). In these calculations we used at minimum $N=4$. However, we observed negligible changes in $P_{\text {ion }}(t)$ up to 


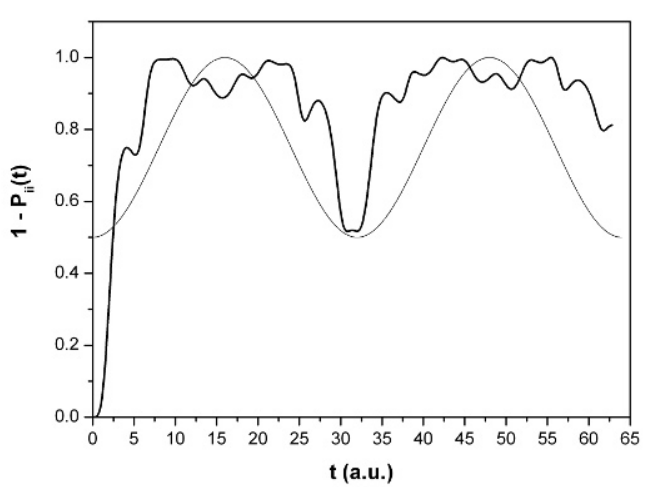

Figure 9. Dependence of probability $1-P_{i i}(t)$ for pulse with $\varepsilon=$ 3 a.u.

$N=7$. The results for the ionization probability are shown in Fig. 10. The oscillations can be distinguished and the period $T_{R}$ is clearly visible. Similar results are obtained for the parameter $\varepsilon=2$ a.u. In addition, since the ionization probability for $\varepsilon=3$ a.u. shows many features, this is a good opportunity to check the approximation (11). Thus, we considered the first three positive energies with even parity corresponding to the "continuum":

$$
\begin{aligned}
& \epsilon_{1}=0.0003 \text { a.u., } \\
& \epsilon_{3}=0.0076 \text { a.u., } \\
& \epsilon_{5}=0.0162 \text { a.u. }
\end{aligned}
$$

Then, according to (11), $\epsilon_{1,3,5}$ can be represented as a narrow band with the appropriate even wave function. This is shown in Fig. 11. Then, we calculated the transition probability

$$
P_{i c}(t)=P_{i \epsilon_{1}}(t)+P_{i \epsilon_{3}}(t)+P_{i \epsilon_{5}}(t)
$$

and compared it with $P_{i c}(t)$ corresponding to (11). The results are shown in Fig. 12. It is clear that all of the features in the probability are quite well reproduced with (11).

In order to verify further the approach we calculated the ionization probability assuming the electric field pulse

$$
E(t)=E_{0} \sin ^{2}\left(\frac{t}{\tau} \frac{\pi}{2}\right) \sin \omega t
$$

as in Geltman's paper [23]. Thus, the appropriate oneperiod evolution operator for the kicking field is

$$
\bar{U}(n T)=e^{-\frac{i}{\hbar} H_{0} \frac{n T}{4}} e^{\frac{i}{\hbar} \bar{p}_{n} z} e^{-\frac{i}{\hbar} H_{0} \frac{n T}{2}} e^{-\frac{i}{\hbar} p_{n} z} e^{-\frac{i}{\hbar} H_{0} \frac{n T}{4}},
$$

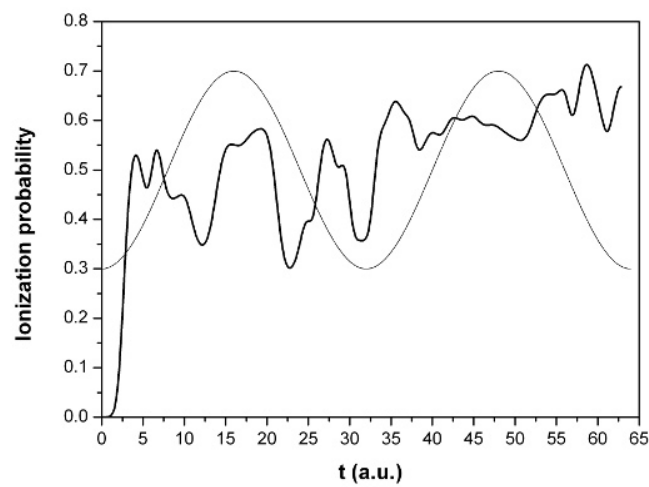

Figure 10. Ionization probability for $\varepsilon=3$ a.u.

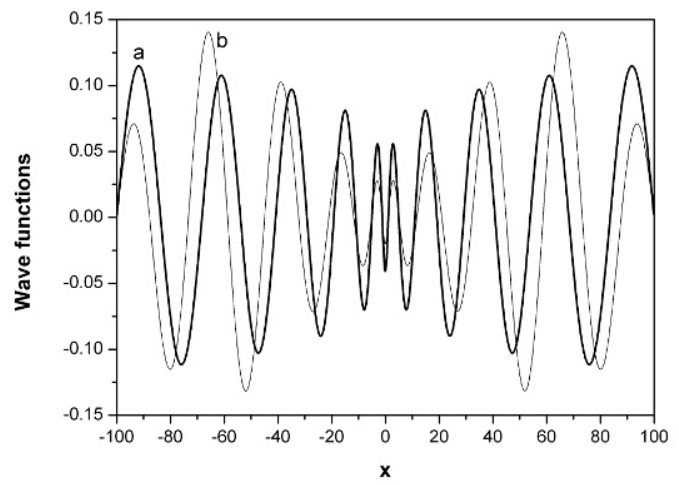

Figure 11. Comparison of the wave function corresponding to $\epsilon_{3}$ (a) with the one obtained with (11) (b).

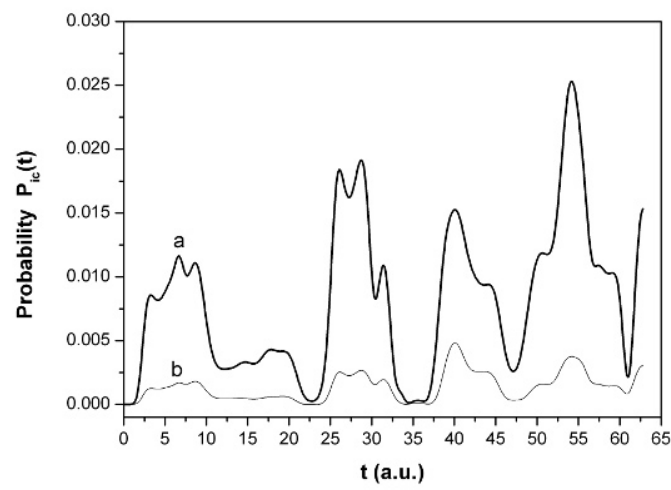

Figure 12. Comparison of the transition probability (53) (a) with the one calculated with (11) (b). 


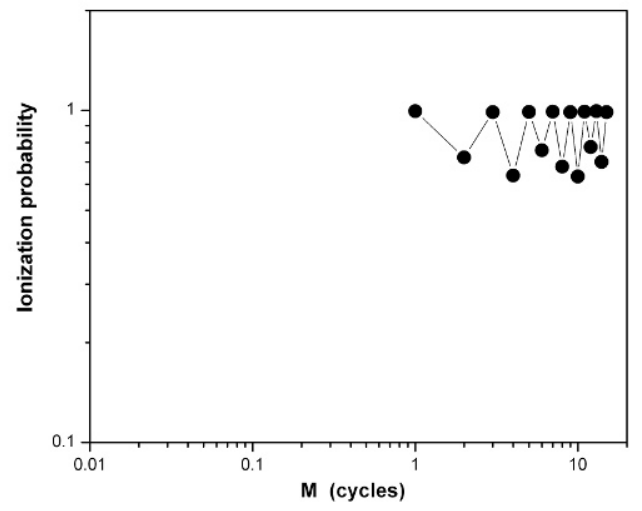

Figure 13. The ionization probabilities for different $M$ values are shown. They can be compared with the appropriate data in Ref. [23] for $E_{0}=1$ a.u. and $\omega=0.55$ a.u.

where $p_{n}=e F_{n} T$ and $\bar{p}_{n}=e F_{n}^{\prime} T$. The $F_{n}$ and $F_{n}^{\prime}$ are obtained from

$$
\frac{1}{T} \int_{0}^{n \frac{T}{2}} E(t) d t=F_{n}, \quad \frac{1}{T} \int_{n \frac{T}{2}}^{n T} E(t) d t=-F_{n}^{\prime},
$$

for $n=1,2, \ldots, M$. The results for $E_{0}=1$ a.u., $\omega=$ 0.55 a.u. and different $M$ values are given in Fig. 13 . They can be compared with the appropriate data given in Ref. [23]. We would like to stress that in the comparison a pulse having integer $\frac{\omega \tau}{2 \pi}$ is assumed. Thus, we have good agreement with Geltman but in our case the oscillations in the ionization probability appear. The analysis of $P_{i f}(n T)$ and $P_{i f}(T)$ in (14) shows that oscillations are intrinsic to the model with unusual kicked dynamics and in this aspect they are artificial. In this approach we use this fact in order to predict a real pulse with a similar effect in ionization probability. In Ref. [23] an electric field pulse in the form

$$
E(t)=E_{0} \sin ^{2}\left(\frac{t}{\tau} \frac{\pi}{2}\right) \cos \omega t
$$

is also considered. By simple analysis it can be shown that this form is not appropriate for our approach. Therefore, we compare (54) and (57) for $\frac{\omega \tau}{2 \pi}=1$. In the first case (sin term) we have $E(\tau)=0$ and

$$
\frac{1}{2 \tau} \int_{0}^{\tau} E(t) d t=-\frac{1}{2 \tau} \int_{\tau}^{2 \tau} E(t) d t \neq 0 .
$$

In the second case (cos term) we have $E(\tau) \neq 0$ and

$$
\frac{1}{2 \tau} \int_{0}^{\tau} E(t) d t=\frac{1}{2 \tau} \int_{\tau}^{2 \tau} E(t) d t=0 .
$$

Thus, a recognized electric field pulse assuming (59) as the corresponding alternating kicking field is impossible. Also, it is difficult to imagine the appropriate $\delta$-shaped pulses at $t=\frac{T}{2}$ and $t=\frac{3 T}{2}$ if the maximum of the electric field pulse is at $t=T$.

\section{Conclusions}

We have presented for study the problem of a $\mathrm{H}(1 \mathrm{~s})$ atom in a spherical box in a pulsed laser field with emphasis on the ionization probability.

The assumption of a spherical box with a radius $a$ simplifies the problem. The boundary condition at $a$ has as a consequence discrete energy spectra of the atomic Hamiltonian in both the negative and the positive parts. In this way we are in the position to analyse completeness of the eigenfunctions of the atomic Hamiltonian and propose the approximate closure relation (24). The unconfined wave functions are used in (24) as an additional approximation making the problem just dependent on $a$. Error is present and we have done everything to estimate and minimize it as it shown in Sec. 3.

The simple model alternating kicking field is taken into consideration. It is demonstrated that minimal error means that in essence we must remain in one-period dynamics. Then, the unusual dynamics of the alternating kicked field is suggested. It is based on recognition of the electric field in the time intervals $[0, T],[0,2 T],[0,3 T], \ldots$ as the corresponding one-period alternating kicking field. Such kick dynamics implicate oscillations in the ionization probability with positive energy states included and participation of the excited states. We have termed these "generalized Rabi oscillations".

With respect to the current interest in this problem the question is how to find the corresponding real pulses which provide such oscillations. We hope that the answer is given in the proposed conditions which establish correspondence with the appropriate dynamics of the alternating kicked field.

The approach is verified in Sec. 4 on the simplified example of a 1D hydrogen atom, and also calculating the previously known results for ionization probability.

\section{References}

[1] J. R. Sabin, E. J. Brandas (Eds.), Theory of Confined Quantum Systems, (Adv. Quantum Chem. 57, 2009)

[2] Y. P. Varshni, J. Phys. B: At. Mol. Opt. Phys. 30, L589 (1997) 
[3] F. P.- Bernal, I. Martel, J. M. Arias, J. G.- Camacho, Phys. Rev. A 63, 052111 (2001)

[4] S. Yoshida et al., Phys. Rev. A 58, 2229 (1998)

[5] M. Rotenberg, Adv. At. Mol. Phys. 6, 233 (1970)

[6] F. B. Dunning et al., Adv. At. Mol. Opt. Phys. 52, 49 (2005)

[7] H. Wiedemann, J. Mostowski, F. Haake, Phys. Rev. A 49, 1171 (1994)

[8] D. Dimitrovski, E. A. Solov'ev, J. S. Briggs, Phys. Rev. Lett. 93, 083003 (2004)

[9] C. Cohen-Tannoudji, B. Diu, F. Laloë, Quantum Mechanics (John Wiley and Sons, New York, 1977)

[10] J. Zhang, T. Nakajima, Phys. Rev. A 75, 043403 (2007)

[11] H. A. Bethe, E. E. Salpeter, Quantum Mechanics of One- and Two-Electron Atoms (Academic Press, New York, 1957)

[12] A. R. Barnett, Com. Phys. Commun. 27, 147 (1982)

[13] L. -Y. Peng, Q. Gong, Com. Phys. Commun. 181, 2098 (2010)

[14] F. P. Bernal, I. Martel, J. M. Arias, J.G.-Camacho, Phys. Rev. A 67, 052108 (2003)

[15] I. J. Thompson, In: F. W. J.Olver et al. (Eds.), NIST Handbook of Mathematical Functions, (Cambridge University Press, 2010)

[16] M. Abramowitz, I. A. Stegun, Handbook of Mathematical Functions, (National Bureau of Standards, Washington, D.C., 1968)

[17] J. S. Dehesa, F. D. Adame, E. R. Arriola, A. Zarzo, In:
C. Brezinski et al. (Eds.), Orthogonal Polynomials and Their Applications, Proc. III International Symposium on Orthogonal Polynomials and Their Applications (June 1990, Erice, Italy)

[18] H. A. Yamani, W. P. Reinhardt, Phys. Rev. A 11, 1145 (1975)

[19] A. T. Stelbovics, J. Phys. B: At. Mol. Opt. Phys. 22, L159 (1989)

[20] E. Bank, M. E. H. Ismail, Const. Approx. 1, 103 (1985)

[21] I. M. Savukov, Phys. Rev. Lett. 96, 073202 (2006)

[22] M. van Faassen et al., Phys. Rev. Lett. 99, 043005 (2007)

[23] S. Geltman, J. Phys. B: At. Mol. Opt. Phys. 33, 1967 (2000)

[24] J. P. Hansen, J. Lu, L. B. Madsen, H. M. Nilsen, Phys. Rev. A 64, 033418 (2001)

[25] H. M. Nilsen, L. B. Madsen, J. P. Hansen, Phys. Rev. A 66, 025402 (2002)

[26] W. Greiner, Quantum Mechanics. An Introduction, (Springer, Berlin, 1994)

[27] M. R. Hermann, J. A. Fleck, Phys. Rev. A 38, 6000 (1988)

[28] L. B. Madsen, Phys. Rev. A 65, 053417 (2002)

[29] A. M. Weiner, Rev. Sci. Instum. 71, 1929 (2000)

[30] J. Javanainen, J. H. Eberly, Q. Su, Phys. Rev. A 38, 3430 (1988)

[31] X. -S. Liu et al., Int. J. Quantum Chem. 79, 343 (2000) 